\title{
New European clinical trial regulations are "unacceptable" to industry
}

Much of the recently proposed European legislation on clinical trials is "unacceptable," says the pharmaceutical industry umbrella organization, the European Federation of Pharmaceutical Industries and Associations (EFPIA; Brussels, Belgium). In a position paper published in December, the EFPIA criticized the draft directive on good clinical practice published by the European Commission in late 1997, saying it would result in "over-bureaucratic requirements," concluding that "Europe may cease to be an attractive location for clinical research."

The draft directive-which is currently being discussed by European Parliamentary committees and which is due to come before the full session of the European Parliament (Strasbourg, France) in April-is intended to implement guidelines drawn up under the tripartite (European Union-Japan-USA) International Conference on Harmonization (ICH) process.

Marie Donnelly, of the European Commission's industry department DGIII, who was heavily involved in drafting the proposals, disputes that it will drive clinical research from Europe. "I don't believe that it is fair to say that bringing Europe into line with international standards is going to

The bureaucratic burden will increase research costs, possibly driving companies outside of Europe. cause research to leave Europe." The directive will achieve a "level playing field" across Europe and the rest of the world, she argues. The reason EFPIA is so voluble on the proposals, she believes, is that they require changes to some European national practices, notably in the UK-Europe's clinical research leader. Specifically, companies conducting phase I toxicity tests on healthy patients currently require the consent of a local ethics committee only. Under the new regulations, however, the UK's Medicines Control Agency (London), for instance, would have to set up a national body to approve phase I trials.

Brian Ager of EFPIA says that the European Commission's directive goes beyond $\mathrm{ICH}$ requirements and in some

Sylvia Davidson is a freelance writer working in London. within 30 days. places contradicts the $\mathrm{ICH}$ guidelines. Three provisions in particular are "unacceptable," according to EFPIA.

First, the provision on clinical safety "contradicts the agreements adopted within the ICH process." While ICH guidelines require only the reporting of serious unexpected adverse events, the proposed directive requires that all serious adverse events in a trial be reported, even if anticipated. Donnelly defends this discrepancy on the basis that many of the European Union's member states "already have the wider requirement and it was difficult to justify reducing [this]". However, she notes that this matter could be reconsidered.

Another bone of contention is the requirements for ethics committees. The new draft directive calls for both national and local ethics committees to approve trial procedures. National committees would have 30 days to give an opinion and the local committees would have 15 days thereafter. EFPIA calls this "complex and conflicting," whereas Donnelly claims that it is the current approval mechanisms in some European countries that are confusing. Local approvals mean that a trial protocol can be changed at each site with the result that a multicenter study can "lose comparability and consistency," she says. The EFPIA suggests that consistency could be achieved if the national and local ethics committees worked in parallel and gave a combined opinion

Another provision the EFPIA deems unacceptable is changes to requirements for manufacturing authorization. Under the directive, investigational products will be subject to the same good manufacturing practice controls as marketed products. EFPIA regards this as impractical at the earli- est experimental stages of a drug's development. Donnelly concedes there may be "a small element of bureaucracy," but she denies that it represents "a heavy burden."

Some of the EFPIA's concerns are shared by nonprofit clinical trials organizations and by patient groups. Denis Lacombe, head of the Investigational Agents Unit of the European Organization for the Research and Treatment of Cancer (EORTC; Brussels, Belgium), believes that the bureaucratic burden will increase the costs of research, possibly driving companies outside of Europe.

If clinical research is no longer done in Europe, it may be difficult for European physicians to become familiar with new treatments, he says. Furthermore, nonprofit organizations may find the costs hard to bear, leading in the long-term, according to Lacombe, to patient suffering. Alastair Kent of the patient body, the Genetic Interest Group (London, UK), agreed that the directive as drafted could make it more difficult to conduct clinical trials.

A further area of concern in the directive is the proposal to establish a database of clinical trials. This could threaten commercial confidentiality or patient confidentiality, according to industry and patient groups. However, Kent also sees the advantages of such a database: "If you do have a database with common diagnostic criteria and accepted standards for rare disorders it does become much more technically feasible to undertake clinical trials." Donnelly understands the industry's concerns, but thinks that the public health argument-that the database will reduce the duplication of trials-should be considered.

Lacombe thinks that resolving all the issues in each European country will take so much time that the non-European arms of a multinational trial will be completed before the European arms begin. "There will be almost no need for Europe to be part of it at all." he says.

Sylvia Davidson 